NASA Technical Memorandum 87333

AIAA-86-1876

19860020158

\title{
Modeling the Effects of Wind Tunnel Wall Absorption on the Acoustic Radiation Characteristics of Propellers
}

Kenneth J. Baumeister Lewis Research Center Cleveland, Ohio

and

Walter Eversman

University of Missouri

Rolla, Missouri

\section{FOR REFERENCE}

Prepared for the

Tenth Aeroacoustics Conference

NOT TO DE TAZEN FHON TUB BOOK

sponsored by the American Institute of Aeronautics and Astronautics

Seattle, Washington, July 9-11, 1986 
AIAAA Paper 86-1876

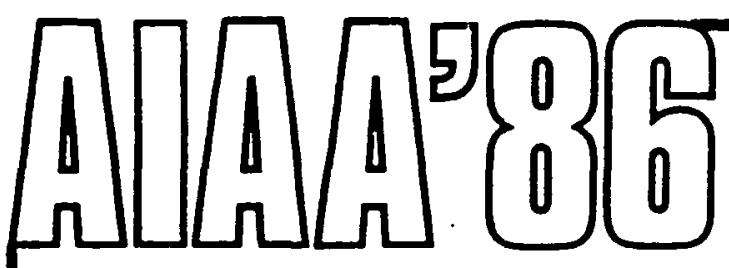

AIAA-86-1876

Modeling the Effects of Wind Tunnel Wall

Absorption on the Acoustic Radiation

Characteristics of Propellers

Kenneth J. Baumeister, NASA Lewis

Research Center, Cleveland, $\mathrm{OH}$;

Walter Eversman, University of Missouri,

Rolla, MO

\section{LIBRARY SOPY \\ -? I I 1986 \\ LANGLEY RESEARCH CENIEK \\ LIERARY, NASA \\ LANGLEY STATION \\ HAMPTON VIRGINIA}

\section{AIAA 10th Aeroacoustics Conference July 9-11, 1986/Seattle, Washington}

For permission to copy or republish, contact the American Institute of Aeronautics and Astronautics 1633 Broadway, New York, NY 10019 
MODELING THE EFFECTS OF WIND TUNNEL WALL ABSORPTION ON THE ACOUSTIC

\title{
RADIATION CHARACTERISTICS OF PROPELLERS
}

\author{
Kenneth J. Baumeister \\ Nationar Aeronautics and Space Administration \\ Lewis Research Center \\ Cleveland, Ohio 44135
}

and

Walter Eversman

University of Missouri

Rolla, Missouri 65401

\section{Abstract}

Finite element theory is used to calculate the acoustic field of a propeller in a soft walled circular wind tunnel and to compare the radiation patterns to the same propeller in free space. Parametric solutions are presented for a "Gutin" propeller for a variety of flow Mach numbers, admittance values at the wall, microphone position locations, and propeller to duct radius ratios. Wind tunnel boundary layer is not included in this analysis. For wall admittance nearly equal to the characteristic value of free space, the free field and ducted propeller models agree in pressure level and directionality. In addition, the need for experimentally mapping the acoustic field is discussed.

\section{Introduction}

The relatively high fuel economy available from propeller-driven aircraft has renewed interest in high speed, highly loaded, multiple blade turboprop propulsion systems. The undesirable features of community noise and, more importantly, the high intensity cabin noise associated with the propellers supersonic helical tip speeds have stimulated new theoretical and experimental research on the acoustic characteristics of turboprops.

The acoustic testing of propellers at realistic inflow Mach numbers can be carried out in flight using a suitably scaled model, or performed in a wind tunnel capable of producing high subsonic flow velocities, again using a scale model. Since the cost of flight testing is high, acoustic testing with supersonic helical tip speed propellers has initially been carried out in the 8 by $6 \mathrm{ft}$ transopic wind tunnel at the Lewis Research Center. $1-6$ Figure 1 displays typical propeller models mounted in the Lewis 8 by 6 wind tunnel. The propeller is driven at the desired speed by an air drive. Noise measurements are made with pressure transducers installed flush with the tunnel walls, through the bleed holes shown in the photograph or on added structures such as the boundary layer refraction plate. As seen in $\mathrm{Fig.} 1$, the hard tunnel wall, wings, or refraction plates could produce significant reverberation effects; thus, the accuracy of the noise measurements has been questioned. In measurements of this type, the test site is essentially a duct containing a noise source represented by the propeller. It is not clear that this test environment will generally produce results for directivity or amplitude which have any relationship to flight test results, although some initial flight to wind tunnel comparisons with the NASA Dryden Jet star aircraft 7,8 have indicated reasonable agreement. Dittmar ${ }^{9}$ has qualitatively arqued that flow convection and the highly directional nature of a typical propeller noise source will minimize reverberation effects when the tunnel Mach number is greater than 0.6. Eversman and Baumeister 10 employed a finite element model of a propeller in a hard wall wind tunnel environment. They found a strong resemblance between the acoustic directivity on the tunnel wall and the general shape of the free field directivity for side line angles of $45^{\circ}$ to $135^{\circ}$. For side line angles less than $45^{\circ}$ or greater than $135^{\circ}$, similarity between duct directivity and free field could not be expected. For a simple monopole and dipole source in a three-dimensional tunnel, Baumeister (Ref. 11) found similar results.

In contrast to the hard wall configurations considered theoretically in Refs. 9 to 11 , in a typical transonic tunnel, sound absorption will occur in the wall bleed holes, which are shown in Fig. 1. Thus, reverberation effects will be reduced and the free field and tunnel response of propellers could have similar directionality and magnitude. The present investigation will use finite element theory to calculate the acoustic field of a propeller in a soft walled wind tunnel and compare these results to the radiation patterns of the same propeller in free space. The absorbing characteristics of the wind tunnel walls are modeled by introducing an admittance boundary condition into the previous hard wall model presented in Ref. 10. The tunnel boundary layer is not considered in this analysis. Thus the shear layer sound refraction effect, known to be large at high Mach numbers for radiation upstream of the propeller, is not considered.

First, the appropriate governing wave equation and boundary conditions will be presented and solved by the finite element method. Next, a parametric study is presented for a number of propeller configurations, wall admittance conditions, and tunnel Mach numbers. Finally, some important results of the parametric study are highlighted and conclusions drawn.

Model for the Propeller in the Wind Tunnel

The propeller in the wind tunnel test environment is modeled using a Gutin propeller representation 12 in a circular duct of uniform cross section. The three-dimensional character of the acoustic field of a propeller in the circular geometry is much more economically modeled than in a rectangular cross section. It is believed that general conclusions drawn by using this simpler 
geometry will be fundamentally the same for the other geometries.

In the Gutin representation, the propeller is replaced by rotating dipoles in the propeller disk. In this section, the governing acoustic equations are presented for an acoustic analogy based on a dipole distribution in cylindrical coordinates. The circular wind tunnel geometry is shown in Fig. 2 , as is the cylindrical coordinate system which is appropriate for this analysis.

The mathematical model has been given in detail in another paper ${ }^{3}$ and the results are briefly repeated here. In terms of nondimensional quantities the propagation of harmonic acoustic disturbances in a circular duct with hard walls is governed by the convected wave equation with the time dependance removed

$$
\nabla^{2} p-\left(i n_{R}+M \frac{\partial}{\partial x}\right)^{2} p=\nabla \cdot \vec{f}
$$

or

$$
\nabla^{2} p-2 i \eta_{R} M \frac{\partial p}{\partial x}-M^{2} \frac{\partial^{2} p}{\partial x^{2}}+\eta_{R}^{2} p=\nabla \cdot \vec{f}
$$

Here $x$ is the axis of symmetry in the cylindrical coordinate system and

$$
\nabla=\frac{\partial}{\partial x} \bar{e}_{x}+\frac{\partial}{\partial r} \bar{e}_{r}-i \frac{m}{r} \bar{e}_{\theta}=0
$$

with $m$ being the angular mode number in an assumed solution of the form

$$
P(x, r, t)=p(x, r) e^{i\left(n_{R} t-m_{\theta}\right)}
$$

Where, consistent with a Gutin type propeller theory, the acoustic analogy representing the propeller requires a distribution of body forces with no volume sources (these would be required if blade thickness were to be modeled). The nondimensionalization begins with the dimensional pressure $p^{\star}$, density $\rho^{\star}$, and velocity $\vec{\nabla}^{\star}$ and introduces their nondimensjonal equivalents; where pressure is scaled by $\rho_{0} c^{2}$, density by $\rho_{0}$, and velocity by $c$. $\rho_{0}$ and $c_{0}$ are reference values of the fluid density and speed of sound. In addition, lengths are scaled by $R$, the tunnel radius, time is scaled by $R / C$, and the body force $f$ (dipole forcing term) scaled by $c^{2} / R$. The mean flow Mach number is $M$ and the nondimensional frequency is

$$
n_{R}=\omega R / c
$$

where $\omega$ is the driving frequency.

Equation (1) or (2), with a suitable body force distribution, represent the classical Gutin theory, as modified for uniform flow effects by Garrick and Watkins. 14 In the case of radiation of propeller noise to a free field, solutions are obtained in a cylindrical coordinate system with a far field radiation boundary condition. When the propeller is inside a circular duct with hard walls a boundary condition of zero normal acoustic velocity (or, equivalently, zero normal pressure derivative) must be enforced at the duct wall.
Equation (1) or (2) are thus supplemented by the condition

$$
\frac{\partial p}{\partial r}=0, \quad r=1
$$

However, when the duct is acoustically lined, as considered herein, the duct-wall boundary condition is characterized by

$$
P=Z V_{n} \text { or } \quad V_{n}=A P
$$

where $V_{n}$ is the particle velocity at the boundary of the in the acoustic lining (assumed in the direction of the outward normal $n), Z$ is the dimensionless specific acoustic $i$ mpedance $\left(Z^{\star} / p_{0} C_{0}\right)$ and $A$ is the acoust ic admittance defined as the reciprocal of specific acoustic impedance. The continuity of the particle displacement gives for the soft wall boundary condition

$$
\frac{\partial P}{\partial r}=i n_{R}\left(1-\frac{i M}{n_{R}} \frac{\partial}{\partial x}\right)\left(1-\frac{i M}{n_{R}} \frac{\partial}{\partial x}\right) A P
$$

The duct is modeled as infinite in length requiring that the "terminations" at $x=0$ and $x=L$ be reflection free. This is most easily enforced by requiring that at the duct terminations the acoustic propagation is given in terms of outgoing acoustic modes (see Ref. 10 for details).

Equation (2) and the appropriate boundary conditions have been solved by Finite Element Theory using the method of Weighted Residuals. In cylindrical coordinates (Fig. 2) the weighted residual statement for the propeller in the wind tunnel has been shown to be 13

$$
\begin{aligned}
& \iint_{S}\left[\nabla W_{i} \cdot\left(\nabla p-M^{2} \frac{\partial p}{\partial x} \vec{e}_{x}-\neq\right)+2 i n_{R} M W_{i} \frac{\partial p}{\partial x}\right. \\
& \left.-n_{R}{ }^{2} W_{i} p\right] r d r d x-\int_{C} W_{i}\left[\nabla p-M^{2} \frac{\partial p}{\partial x} \vec{e}_{x}\right] \cdot n d S=0
\end{aligned}
$$

for all weighting functions $W_{j}$ contained in a suitable class of functions. The surface $S$ is the $x, r$ plane in cylindrical coordinates and $C$ is the boundary of the computational domain consisting of the tunnel termination planes and the tunnel walls.

The soft wall boundary condition is also imposed through the boundary residual. The requirement that for a point reacting liner of nondimensional admittance $A$ to the condition at the boundary $r=1$, yields the boundary integral

$$
\begin{aligned}
\int_{C} w_{i}[\nabla p & \left.-M^{2} \frac{\partial p}{\partial x} e_{x}\right] \cdot \vec{e}_{r} d x=\int_{C} w_{i} \frac{\partial p}{\partial r} d x \\
& =i n_{R} \int_{C} w_{i}\left(1-i \frac{M}{n_{R}} \frac{\partial}{\partial x}\right)\left(1-i \frac{M}{\eta_{R}} \frac{\partial}{\partial x}\right) A p d x
\end{aligned}
$$

In order to reduce the continuity requirements on the solution, and to reduce the discontinuity introduced by jumps in the lining admittance, an 
integration by parts is carried out on the boundary term to replace Eq. (10) by

$$
\begin{gathered}
\int w_{i} \frac{\partial p}{\partial r} d x=-i n_{r} \int_{1}^{2} w_{i} A p d x-2 M \int_{1}^{2} A w_{i} \frac{\partial p}{\partial x} d x \\
-i \frac{M^{2}}{n_{R}} \int_{1}^{2} A \frac{\partial W_{i}}{\partial x} \frac{\partial p}{\partial x} d x-2 M \sum_{n=1}^{N} w_{i}\left(x_{n}\right) p\left(x_{n}\right) \Delta A_{n} \\
-i \frac{M^{2}}{n_{R}} \sum_{n=1}^{N} \frac{\partial w_{i}}{\partial x}\left(x_{n}\right) p\left(x_{n}\right) \Delta A_{n}
\end{gathered}
$$

in which $x_{n}$ are points between 1 and 2 where the lining admittance changes by $\Delta A_{n}$. The terms additional to the integral account for discontinuities in admittance which are significant when a mean flow is present.

This model has been used to investigate the effect of having an acoustic lining on the wind tunnel outer wall for the purpose of creating an approximate free field environment. These model equations have been solved by finite element theory which has been well documented in Ref. 13.

\section{Model for the Propeller in the Free Field}

In the case of radiation of propeller noise to a free field, the same governing equations apply as in the tunnel; however, the far field radiation boundary condition is applied around the boundary of the computational domain and the characteristic frequency is now defined in terms of the propeller radius. In this case, the orientation of the elements are radial in nature as shown by the dashed pie shape lines in Fig. 3. In contrast, dashed rectangular shape cuts in Fig. 4 represent the element shapes used to model the acoustic field in the tunnel. As with the in-tunnel calculations, details of the finite element solution can be found in Ref. 13.

\section{Propeller Frequency Parameters}

Equation (5) defined the dimensionless acoustic frequency $n_{R}$ of the wind tunnel in terms of the tunnel radius $R$, driving acoustic frequency $\omega$ and the speed of sound. This frequency can be related to the propeller shaft angular velocity $\Omega(\mathrm{rad} / \mathrm{sec})$ and the number of propeller blades $N$ by the relationship

$$
\omega=N \Omega n
$$

where $n$ is the narmonic number. Thus

$$
n_{R}=\frac{R \omega}{C}=\frac{R N \Omega n}{C}
$$

The velocity ratio of the propeller tip to sonic velocity is defined as blade Mach number and is equal to

$$
M_{B}=\frac{V_{p}}{C}=\frac{R_{p} \Omega}{C}
$$

When $R_{p}$ s equals the sonic velocity $c$, then $M_{B}$ equals unity. In addition to $n_{R}, a$

propeller dimensionless acoustic frequency can now be defined in terms of the velocity ratio as follows

or

$$
n_{p}=\frac{R_{p} \omega}{c}=\frac{R_{p} \Omega N n}{c}
$$

$$
n_{p}=M_{B} N n
$$

Recall, the angular mode number $m$ appears in the governing equation along with $n_{R}$ through the operator $\nabla$ in Eq. (4). The mode number $m$ can be related to the blade number $N$ through the standard equation

$$
\mathrm{m}=\mathrm{Nn}-\mathrm{KN} \mathrm{V}
$$

where $N_{V}$ represents the number of guide vans or disturbances and $K$ is an integer constant. For our case, in the absence of vans or disturbances

$$
\mathrm{m}=\mathrm{Nn}
$$

and Eq. (16) simplifies to

$$
n_{p}=M_{B} m
$$

The effective propeller frequency is the key parameter of a propeller in a free field environment. The tunnel acoustic frequency can now be redefined as

$$
n_{R}=n_{p}\left(R / R_{p}\right)
$$

Thus, for a fixed dipole distribution, the propeller acoustic frequency $n p$, radius ratio $R / R_{p}$, angular mode number $m$ and flow Mach number set the important parameters for a particular calculation. For example, for the first harmonic $(n=1)$ of a six bladed propeller $(N=6)$ operating with sonic tips $\left(M_{B}=1\right)$, the dimensionless acoustic propeller frequency of $n_{\mathrm{p}}$ would be 6 . For a tunnel to propeller radius ratio of $2, n_{R}$ would be 12 .

\section{Parametric Calculation}

Finite element solutions will now be presented for a number of propeller acoustic frequencies, flow Mach numbers, wall admittance, propeller to duct radius ratio, and blade numbers. From these parametric calculations some conclusions will be drawn concerning the nature of testing a propeller in a transonic wind tunnel.

\section{No Flow Example}

Figure 3 shows the directivity for a propeller in a free field environment at $M=0.0$. This is a six-bladed propeller $(N=6)$ rotating at $M_{B}=1$, which corresponds to a tip speed of sonic velocity. The fundamental harmonic $(n=1)$ is considered, which according to Eq. (18) creates an angular mode number of $m=6$, and according to Eq. (19) produces a dimensionless propeller acoustic frequency of $\mathrm{np}=6$.

Figure 3 displays contours of equal acoustic pressure amplitude in a plane of constant $\theta$ in 
the cylindrical coordinate system. As expected in a comparison to classical "Gutin" theory, the directivity is asymmetric with the maximum Sound Pressure Level occurring behind the propeller. Two distinct lobes are present and an SPL minimum just ahead of the propeller. The first question to be addressed is how the directivity would be altered if this propeller is operated in a hard wall tunnel.

Figure 4 shows a similar plot for the same propeller in a circular wind tunnel with an outer radius twice the propeller radius, and again with no mean flow. The nondimensional acoustic frequency based on the duct radius is $n_{R}=12$, according to Eq. (20). In Fig. (4), no acoustic lining is present and the boundary condition designated by Eq. (6) is enforced on the surface of the elements adjacent to the tunnel walls. The pressure amplitude contours upstream in Fig. 6 are lower than the contours downstream, and fall below minimum level plotted.

Near the propeller, the radiation pattern is similar to the free field directivity. However, at the duct walls, where measurements may be conveniently made, 1 the presence of the wall considerably modifies the acoustic field. At both large positive and negative axial distance from the propeller plane, the classic acoustic duct mode patterns are displayed. In these regions distant from the propeller source, no similarity between the propeller in a free environment could be expected. Even in the propeller plane evidence of a standing wave pattern is seen.

In Fig. 5, an acoustic lining with admittance $A=0.5+\mathfrak{i} 0.0$ is used at the tunnel walls. In this case, the radiation pattern develops lobes much more representative of the free field pattern. Although standing wave patterns are again seen away from the propeller. This shown more more conclusively in Fig. 6 where the directivity is plotted at the wall in the wind tunnel case and at the equivalent side line positions in the free field case. In Fig. 6, a comparison is made of the free field directivity and the directivity in the wind tunnel with several linings and in the unlined (hardwall) case.

A dramatic improvement of the directivity on the duct wall is seen when the duct is lined. With $A=0.5+i 0.0$, the peak levels are matched and the in-duct and free field directivities are similar. For a larger value of admittance $(A=1.0+$ $i 0.0)$, the pressure level can fall below the free field value.

\section{High Velocity Flow Example $(M=0.5)$}

A comparison at $M=-0.5$ for a two-bladed propeller at a dimensionless propeller frequency of $n_{p}=2.0, m=2$, and duct frequency $n_{R}=4.0$ is shown in Figs. 7 to 10 . Figure 7 displays the free field radiation pattern. Figure 8 gives the radiation pattern and directivity in the hard wall duct and Fig. 9 presents the duct radiation pattern with wall admittance $A=0.5+i 0.0$. This admittance produces duct radiation patterns which match free field results upstream of the propeiler as shown in Fig. 10. These results are similar to the case without flow in that the lining enhances the reproduction of the free field directivity in the tunnel.

In Fig. 10, the free field and in-duct directivities are compared for several lining admittances. In the axial vicinity of the source, hard wall tunnel directivity and magnitude are in surprisingly close agreement. However, the classic hard wall $6 \mathrm{~dB}$ correction was not applied to these results. This result may be fortuitous as will be discussed later. To obtain agreement for $|x / R|>0.25$ the walls must be lined. For a wall admittance of $A=0.5+i 0.0$, the amplitude and directivity of the pressure on the wall downstream of the propeller is in excellent agreement with the free field values. However, this is not the case upstream of the propeller.

When sound propagates against the flow as compared to with the flow, the wall admittance must be set at a different value to obtain uniform suppression in the tunnel. Thus, at relatively high Mach numbers, the walls should be constructed with different admittance values upstream and downstream of the propeller.

\section{Low Velocity Flow Example $(M=-0.2)$}

A comparison between free field and wind tunnel acoustic radiation characteristics for a eight-bladed propeller at a dimensionless propeller frequency of $n p=5.69, m=8, n=1$, and duct frequency 11.38 is shown in Figs. 11 to 13 with $M=-0.2$. This Mach number coincides with the landing speed of a turboprop aircraft. In this case, community noise must be considered; consequently, accurate predictions of the far field noise are important.

Figure 11 displays the free field radiation pattern as well as the radiation pattern for the same propeller in hard and soft wall tunnel configurations. As seen in Fig. 11(b), a hard wall tunnel leads to equal pressure lobes fore and aft of the propeller which is in contrast to the free field result where the rear lob dominates. Increasing the wall admittance improves the modeling of the free field propeller. The admittance of $0.91+\mathbf{i 0 . 0}$ (approximately free field impedance $\rho_{0} c_{0}$ ) seems to give the best agreement. Notice in Fig. 11(e), that making the wall softer distorts the radiation pattern such that lobes are no longer present.

In Fig. 12, the free field and in-duct directivities are compared for several lining admittances. Again the results for this low Mach number case are similar to the no flow and high velocity cases in that the lining enhances the reproduction of the free field directivity in the tunnel. However, in contrast to the high velocity case shown in Fig. 10, in the vicinity of the source, hard wall tunnel directivity and magnitude do not match the free field characteristic of the propeller. In the admittance range of $0.5+i 0.0$ to $0.91+\mathbf{i} 0.0$, however, the agreement between magnitude and direction are in excellent agreement along the tunnel wall. Unfortunately, theory also shows that it is possible to make the tunnel walls too soft and destroy the comparison between both the magnitude and directivity of the ducted and free field propeller configurations.

Finally, pressure measurement along a soft absorbing wall can be difficult to obtain and interpret. For example, the classic $6 \mathrm{~dB}$ hard wall correction factor can not be applied. Figure 13 displays the directivity and magnitude off the wall at a radius ratio of 1.667 . As seen in Fig. 13, the agreement between the free field and tunne 1 results is slightly improved over that shown in Fig. 12 . 
The NASA Lewis Research Center personnel are performing low speed propeller noise measurements in the return leg of the 8 - by 6 -Foot Transonic Wind Tunnel, see Fig. 14 . The 9- by 15 -Foot return leg tunnel has been lined with acoustic material to obtain free field estimates of propeller noise at 1 anding speeds. the design procedure that specified the acoustic properties as a function of frequency of the Lewis 9- by 15-Foot Wind Tunnel can be found in Ref. 15 .

The previous numerical comparisons in Figs. 1 to 13 conform to the high speed leg of the Lewis tunnel for which the ratio of tunnel to propeller radius of 2 is more appropriate. For the 9- by 15-Foot Tunnel simulations, a tunnel to propeller radius of 6 will be assumed. For the dimensionless propeller frequency $n_{p}=5.69$, the tunnel frequency now becomes $n_{R}=34.13$ according to

Eq. (20). Recall, the duct frequency parameter $n_{R}$ appears in the governing Eq. (9).

A comparison between free field and wind tunnel acoustic vadiation characteristics for a eight-bladed propeller at a dimensionless propeller frequency of $\pi_{p}=5.69, m=8, n=1$, and duct frequency 34.13 is shown in Figs. 15 and 16 $M=-0.2$. In Fig. 15, the free field and in-duct directivities are compared for several lining admittances. As with the low flow case in the small tunnel $\left(R / R_{p}=2\right)$, an admittance value of $0.5+i 0.0$ to $0.91+i 0.0$ gave reasonable propeller directivity patterns near the source.

In this large tunnel, the duct acoustic modes dominate upstream, downstream, and near the tunnel walls. Consequently, the tunnel walls would be an inappropriate place to attempt to determine the far field directionality of a propeller. For this paper, the measurement plane is taken to be at twice the propeller radius, or $r / R$ equal to 0.333 . As seen in Fig. 16, over the whole range of wall admittances, the free field and tunnel measurements are in close agreement in magnitude and direction. Downstream, for $X / R$ greater than 0.3 , however, the duct acoustic modes begin to dominate, while upstream the tunnel results will simulate free field over larger axial distance. At this Mach number, there is no need to tailor the upstream and downstream admittances differently.

\section{Propeller and Duct Physics}

The radiation properties of the propeller source at low Mach number $(-0.2)$ is displaced in Fig. 17. Near the propeller source the field is outwardly radiating and falling off with radial distance $r$. A few wavelengths from the source, the field will begin to decay inversely proportional to radius squared. For a wind tunnel measurements to be valid, ideally the acoustic field in the tunnel should also have these properties.

As has been shown in the directivity plots of Figs. $4,5,8,9,11$, and 15 the contour lines of equal pressure amplitude are quite complex. Figure 18 displays a magnified view of the directivity plot for the $M=-0.2$ case in the large wind tunne 1 configuration $\left(R / R_{p}=6\right)$. An examination of these field lines will now be made to better illustrate limitations on valid comparisons between tunnel and free field propeller acoustics.
Near the propeller (labeled prop in Fig. 18) the contours of equal pressure amplitude radiate outward and the spl level falls with increasing radius. In Fig. 18, this portion of the field is labeled the outward radiation region. Our comparisons with the free field in Fig. 16 showed that similarity in magnitude and direction between the free and duct propeller are excellent. This region is ideally suited for testing the radiative characteristics of propeller models.

The region near the tunnel wall itself is called the pseudo far field regions because of the complicated nature of sound pressure level contours. For example, in the range $X / R$ of 0.0 to 0.2 and $r / R$ of 0.5 to $r / R$ of 1.0 the SPL contour lines are vertical. Clearly, in this region, the SPL lines are almost independent of $r$. Thus, this domain obviously does not simulate the free field characteristics of a propeller.

To further amplify this point, return to the high Mach number problem in the smaller $R / R_{p}=2$ wind tunnel model. Recall in our discussion of Fig. 10, it was stated that the hard wall tunnel directivity and magnitude were in surprisingly close agreement. However, inspection of the directivity of this duct propelier configuration shown in Fig. 8 shows that the entire region of agreement occurs in the pseudo far-field region. Recall the axial directionality plots were along the top wall as were the experimental data reported in Refs. 1 to 9. A close inspection of Fig. 8 for $r / R$ from 0.6 to 1.0 (wall) and $X / R$ from 1.6 to 2.4 shows the SPL contours to be nearly vertical. As a result, at a Mach number of -0.5 , comparison between the free field and a hard wall tunnel can at best be fortuitous. The soft wall case displayed in Fig. 9, however, shows a SPL variation in $r$ near the wall. The NASA high speed ( 8 by $6 \mathrm{ft}$ ) wind tunnel used for the measures reported in Refs. 1 to 9 fortunately has soft wall characteristics. The bleed holes shown in Fig. 1 on the tunnel walls have resistance and reactive properties which lead to nonzero values of admittance $A$.

A third region displayed in Fig. 18 on the left hand side is labeled the duct acoustic region. In this region, the modes associated with duct propagation dominate. The SPL level line can become horizontal indicating constant pressure with axial distance. As a result similarity between duct directivity and free field directionality along a sideline can not be expected.

Finally, a fourth region on the right hand side of Fig. 18 labeled approximate far field region is shown. This region may or may not exit for any particular tunnel because of the source configuration and from reflections due to the change of admittance at the boundary. The usual calibration tests required of an anechoic chamber might be required. Since the constant pressure radial lines of Fig. 17 are absent from Fig. 18, at best the approximate far field region exists over very small portions of the domain shown in Fig. 18 .

Clearly, in performing experiments in wind tunnels with propeller source, experimental mapping of the acoustic field is required.

\section{CONCLUDING REMARKS}

Finite element theory was used to calculate the acoustic field of a propeller in a soft walled circular wind tunnel and to compare the radiation patterns to the same propeller in free space. 
Parametric solutions were presented for a "Gutin" propeller for a variety of flow Mach numbers, admittance values at the wall, microphone position locations, and propeller to duct radius ratios. At low or zero Mach numbers, the wall of the wind tunnel must be lined with acoustic absorbers for the free field and ducted propeller models to have agreement in pressure level and directionality. Best agreement will occur when measurements are made off the tunnel walls and with tunnels having large wall to propeller radius ratios. At high Mach numbers $(M=-0.5)$, for both the free and ducted representations, numerical calculations indicate that in the vicinity of the propeller the pressure level along a side line (tunnel wall) have similar directivity and magnitude for a hard wall and a range of soft wall configurations. However, the hard wall results are most likely fortuitous because the calculations were made in the pseudo far field region of the flow. More theoretical calculations and experimental measurements for a range of high tunnel Mach numbers may be required to resolve this problem.

At larger distance from the source (nearer to the axis, upstream or downstream) soft wall liners can suppress the usual duct modes if the wall admittance values are properly chosen. In this case, the agreement between the free field and duct radiation characteristics will occur over greater sideline distances. At high Mach numbers for the suppression to be uniform on both sides of the propeller, the wall admittance should be tailored differently upstream and downstream of the propeller. At low velocities, the tailoring is unnecessary. However, the wall must not be made too soft (large admittance). In this case, the pressure magnitude could be greatly diminished by the interaction of the transmitted and reflected waves from the wall.

\section{References}

1. Dittmar, J.H., Blaha, B.J., and Jeracki, R.J., "Tone Noise of Three Supersonic Helical Tip Speed Propellers in a Wind Tunnel at 0.8 Mach Number," NASA TM-79046, 1978.

2. Dittmar, J.H., Jeracki, R.J., and

Blaha, B.J., "Tone Noise of Three Supersonic Helical Tip Speed Propellers in a Wind Tunnel," NASA TM-79167, 1979.
3. Dittmar, J.H., Jeracki, R.J., "Additional Noise Data on the SR-3 Propeller," NASA TM-81736, 1981.

4. Dittmar, J.H., Stefko, G.L., and Jeracki, R.J., "Noise of the 10-Bladed, $40^{\circ}$ Swept SR-6 Propeller in a Wind Tunnel," NASA TM-82950, 1982.

5. Dittmar, J.H., Stefko, G.L., and Jeracki, R.J., "Noise of the 10-Bladed, $60^{\circ}$ Swept SR-5 Propeller in a Wind Tunnel, NASA TM-83054, 1983.

6. Dittmar, J.H. and Jeracki, R.J., "Noise of the SR-3 Propeller Model at $2^{\circ}$ and $4^{\circ}$ Angle of Attack," NASA TM-82738, 1981.

7. Mackall, K.G., Lasagna, P.L., Walsh, K., and Dittmar, J.H., "In-Flight Acoustic Results from an Advanced-Design Propeller at Mach Numbers to $0.8, "$ AIAA Paper 82-1120, 1982.

8. Dittmar, J.H. and Lasagna, P.L., "A Preliminary Comparison Between the SR-3 Propeller Noise in Flight and in a Wind Tunnel," NASA TM-82805, 1982.

9. Dittmar, J.H., "Why Credible Propeller Noise Measurements are Possible in the Acoustically Untreated NASA Lewis $8 \mathrm{Ft}$ by $6 \mathrm{Ft}$ Wind Tunnel," Journal of the Acoustical Society of America, Vol. 75, No. 6, June 1984, pp. 1913-1914.

10. Eversman, W. and Baumeister, K.J., "Modeling of Wind Tunnel Wall Effects on the Radiation Characteristics of Acoustic Sources," AIAA Paper 84-2364, 0ct. 1984.

11. Baumeister, K.J., "Reverberation Effects on Directionality and Response of Stationary Monopole and Dipole Sources in a Wind Tunnel," Journal of Vibration, Acoustic, Stress, and Reliability in Design, Vol. 108 , No. 1, Jan. 1986, pp. 82-90.

12. Gutin, L., "On the Sound Field of a Rotating Propel1er," NACA TM-1195, 1948.

13. Eversman, W. and Steck, J.E., "Finite Element Modeling of Acoustic Singularities with Application to Near and Far Field Propeller Noise," AIAA Paper 84-2286, Oct. 1984.

14. Garrick, I.E. and Watkins, C.E., "A Theoretical Study of the Effect of Forward Speed on the Free-Space Sound-Pressure Field Around Propellers," NACA Report 1198, 1953.

15. Dahl, M.D. and Rice, E.J., "Measured Acoustic Properties of Variable and Low Density Bulk Absorbers," ASME Paper 85-WA/NCA-6, Nov. 1985. 

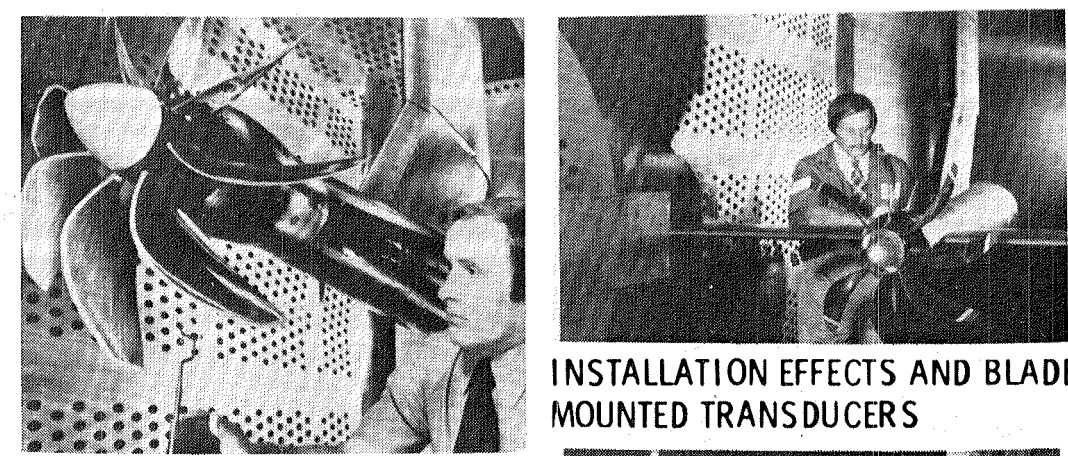

INSTALLATION EFFECTS AND BLADE MOUNTED TRANSDUCERS

PROPELLER ALONE AND AT ANGLE ATTACK

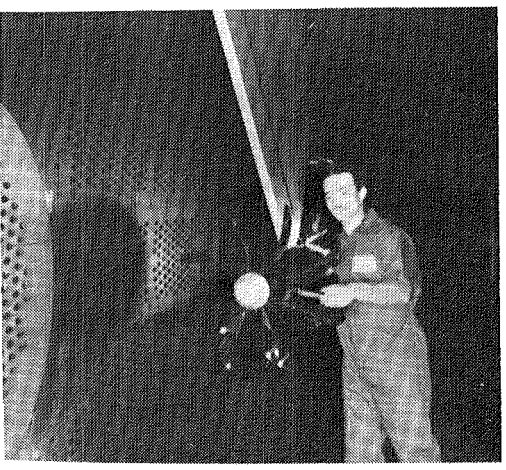

WING SHIELDING

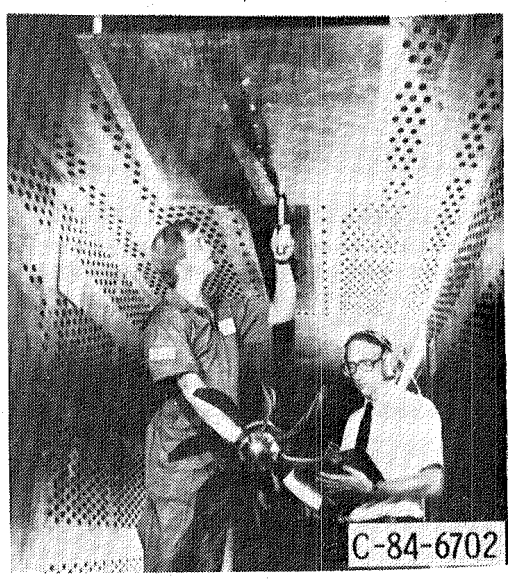

BOUNDARY LAYER REFRACTION

Figure 1. - Advanced prop-fan installations in NASA Lewis Research Center $8 \times 6 \mathrm{ft}$ Transonic Wind Tunnel.

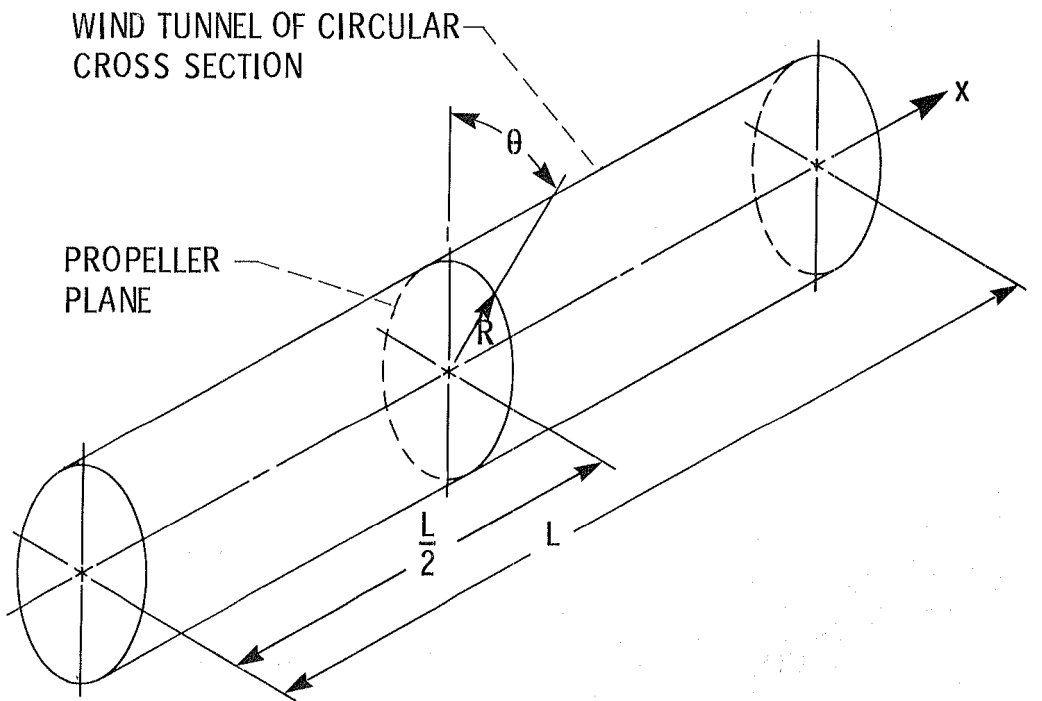

Figure 2. - Geometry of the propeller in a circular wind tunnel. 


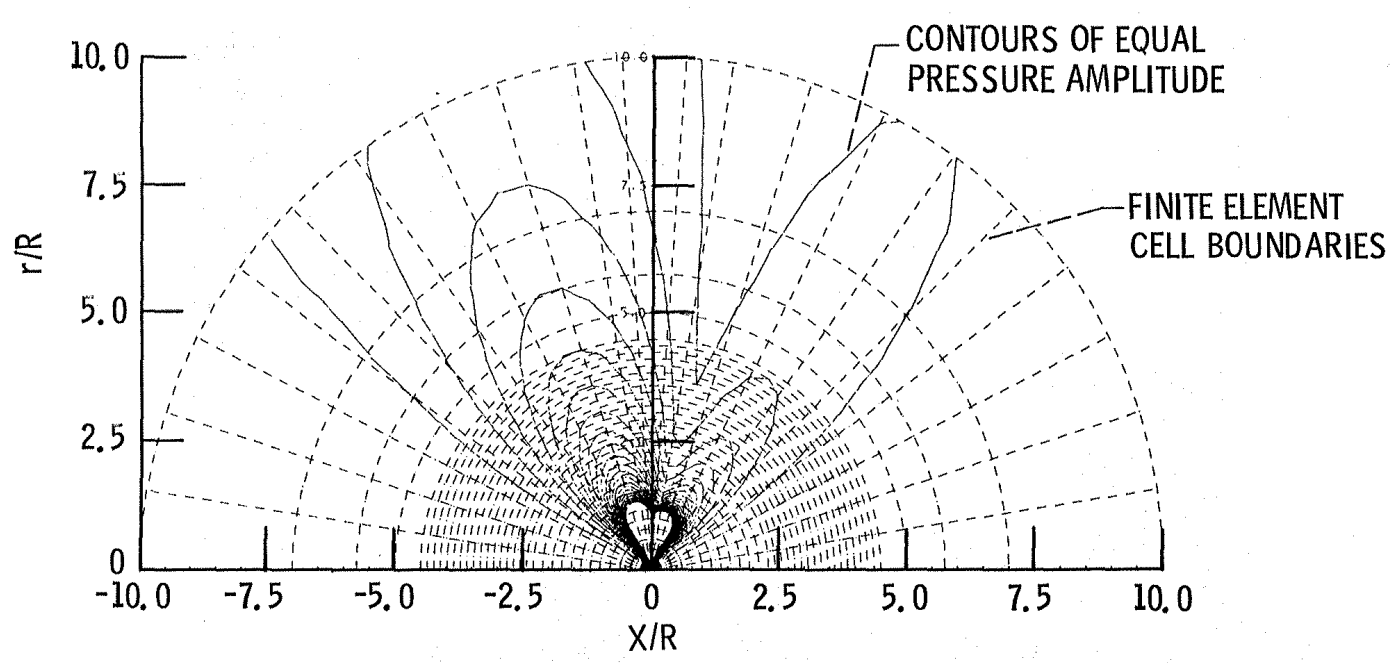

Figure 3. - Free-field directivity for the six-bladed propeller; $m=6$, $\eta_{P}=6, M=0.0$.

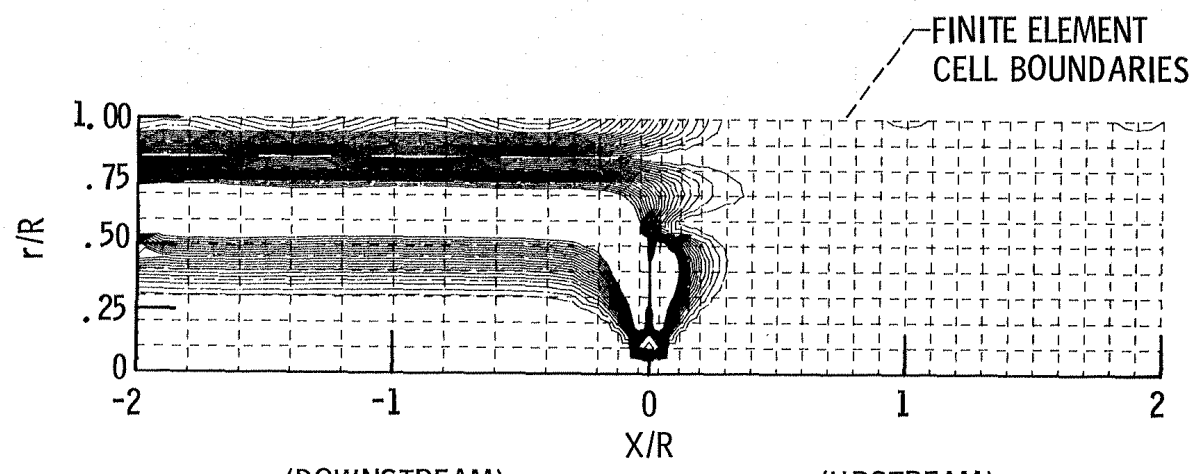

(DOWNSTREAM)

(UPSTREAM)

Figure 4. - Directivity for the six-bladed propeller in the hard walled wind tunnel; $m=6, \eta_{P}=6$ (propeller), $\eta_{R}=12$ (duct), $M=0.0$. (Contours of equal pressure amplitude.)

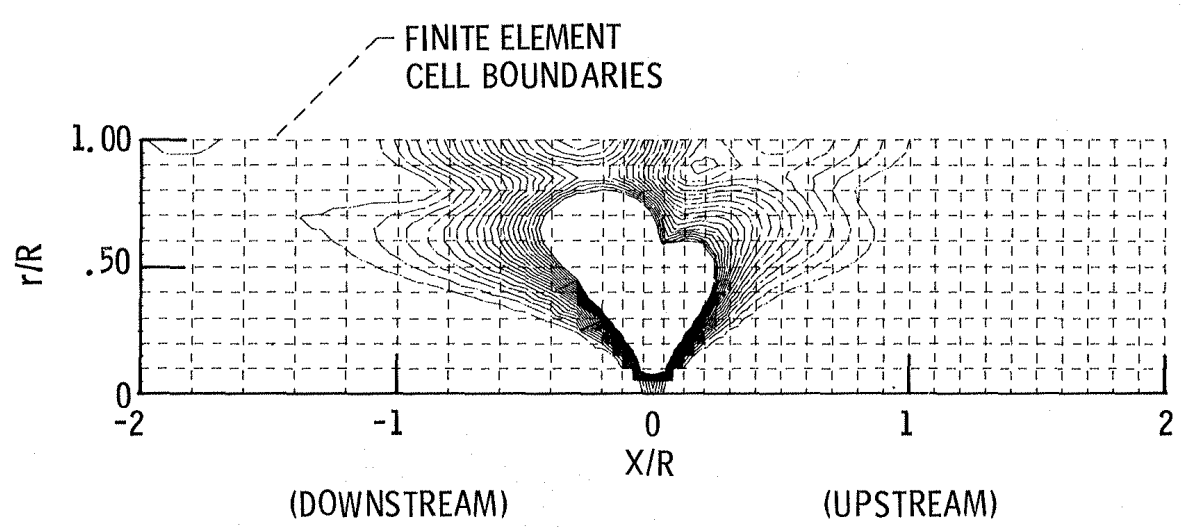

Figure 5. - Directivity for the six bladed propeller in the wind tunnel with wall admittance; $A=0.5+i 0.0, m=6, \eta_{P}=6$ (propeller), $\eta_{R}=12$ (duct), $M=0.0$. (Contours of equal pressure amplitude.) 


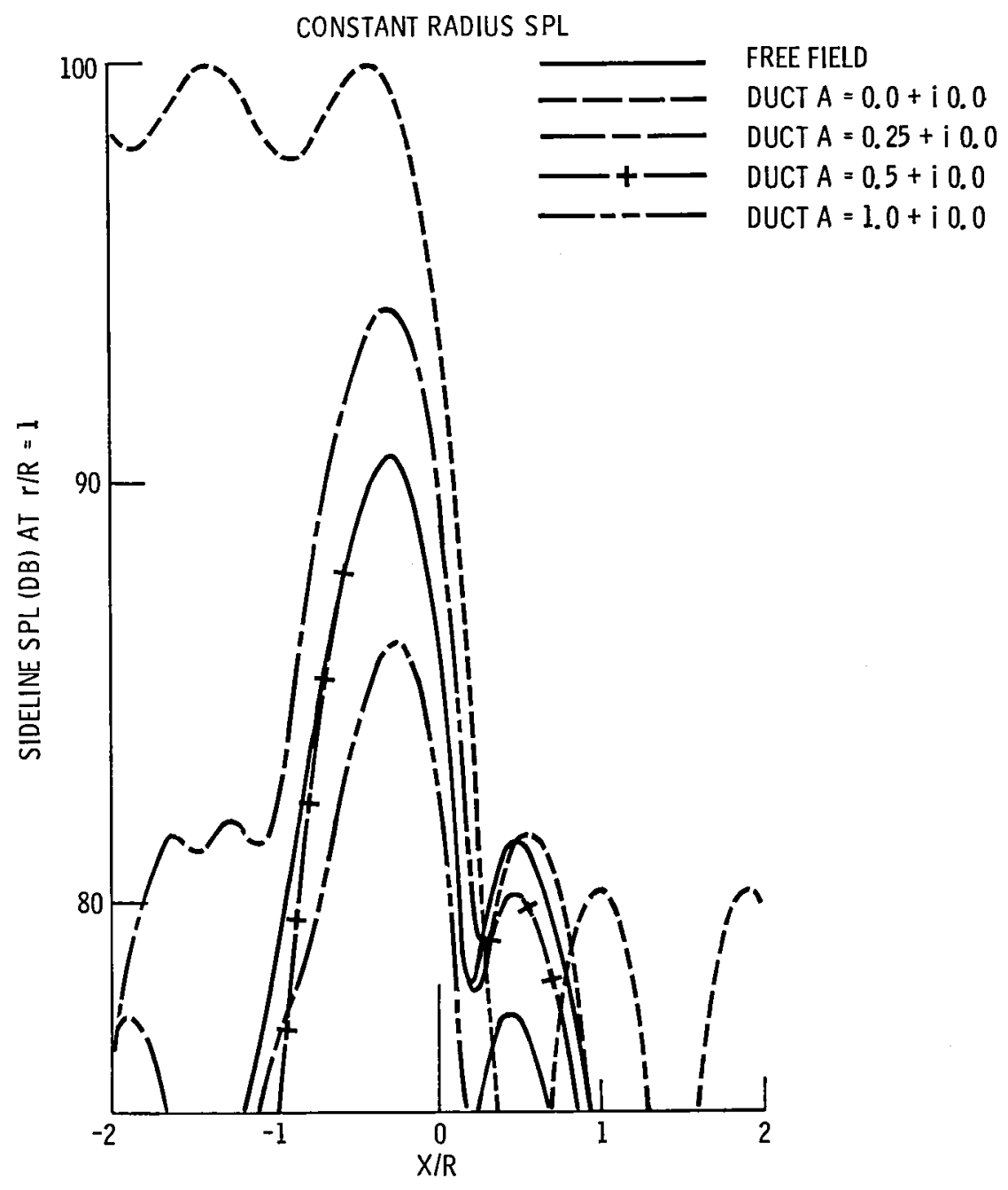

Figure 6. - Sideline directivity at tunnel radius of two propeller diameters $\left(r / R_{p}=2\right.$ or $\left.r / R=1\right)$ comparing the propeller in the free field (solid line) and in the tunnel with several lining admittances; $m=6, \eta_{P}=6$ (propeller), $\eta_{\mathrm{R}}=12$ (tunnel), $M=0.0$.

(AFT) (FORWARD)

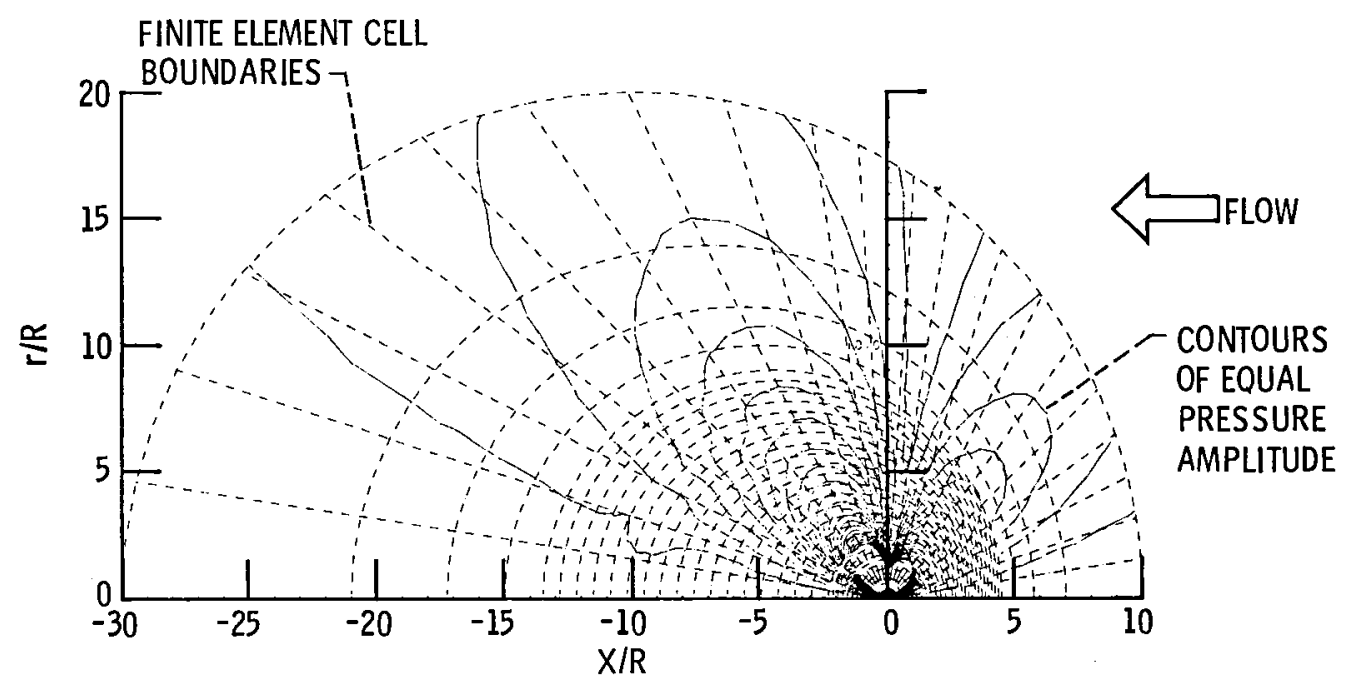

Figure 7. - Free field directivity for the two bladed propeller in a mean flow; $m=2, n_{P}=2, M=-0.5$. 


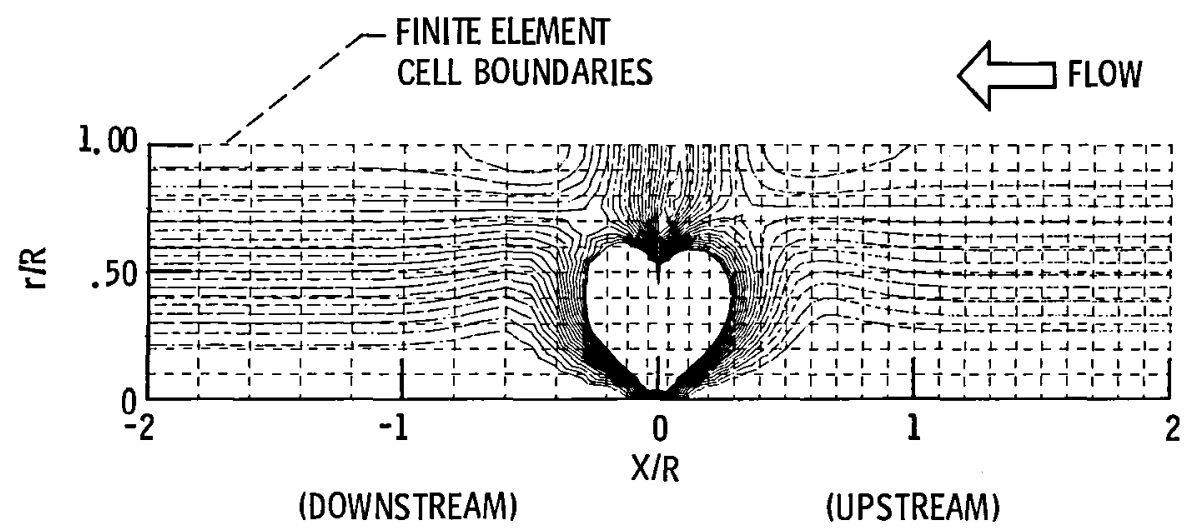

Figure 8. - Directivity for the two bladed propeller in the hard walled wind tunnel with mean flow; $m=2, \eta_{P}=2$ (propeller), $\eta_{R}=4$ (duct), $M=-0.5$. (Contours of equal pressure amplitude. )

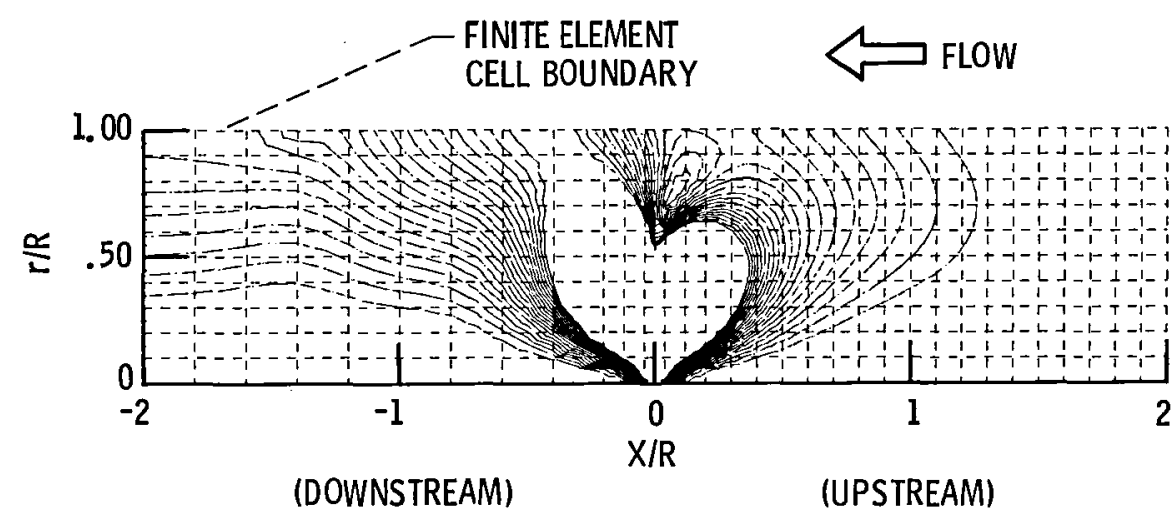

Figure 9. - Directivity for the two bladed propeller in the duct with a wall impedance of $A=0.5+i 0.0$ and with a mean flow; $m=2, \eta_{P}=2$ (propeller, $n_{R}=4$ (duct), $M=-0.5$. (Contours of equal pressure amplitude.) 


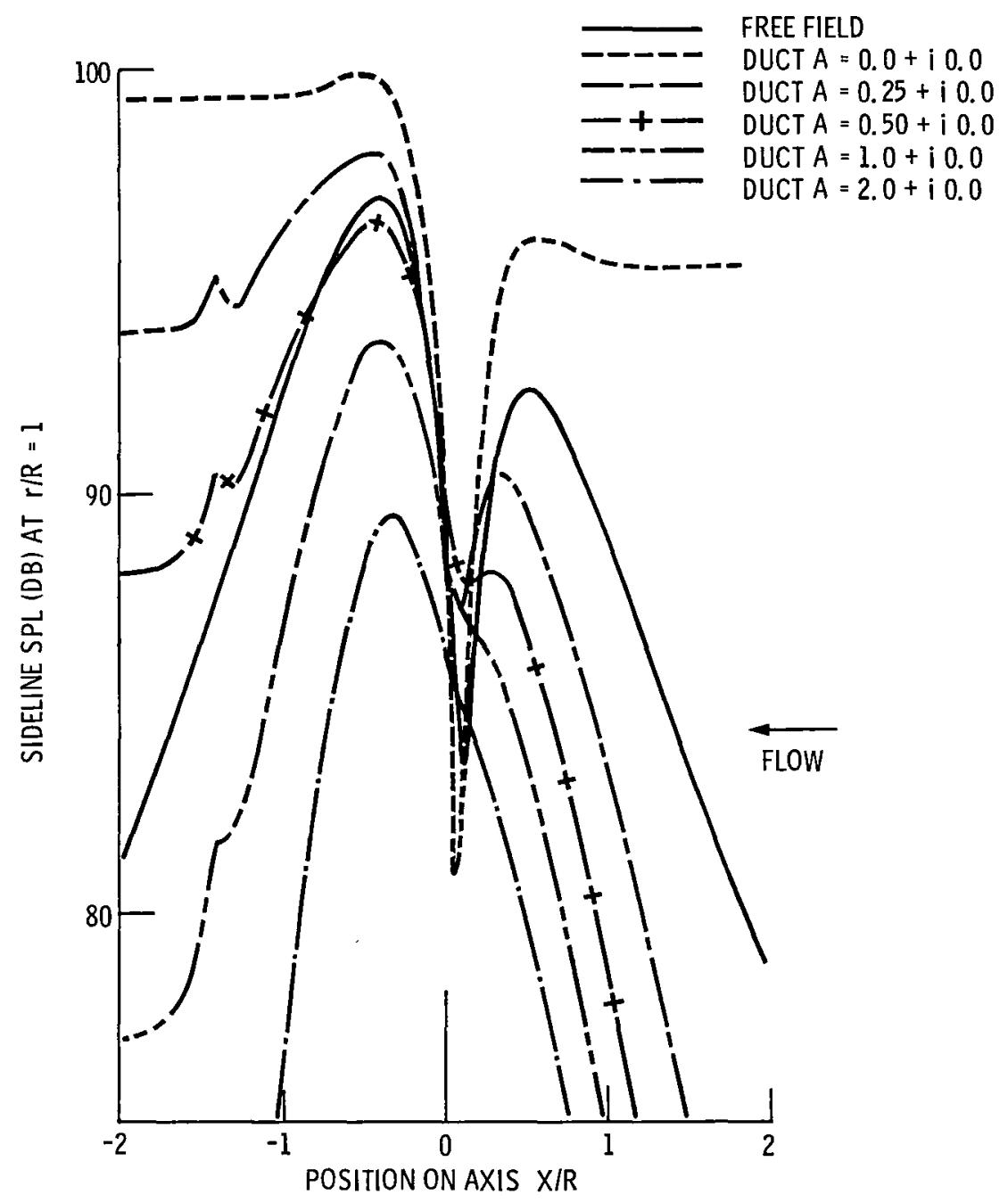

Figure 10. - Sideline directivity at tunnel radius of two propeller diameters $\left(r / R_{p}=2\right.$ or $\left.r / R=1\right)$ comparing the propeller in the free field (solid line) and in the tunnel with several lining admittances; $m=2$, $\eta_{P}=2$ (propeller), $\eta_{R}=4$ (tunnel), $M=-0.5$. 


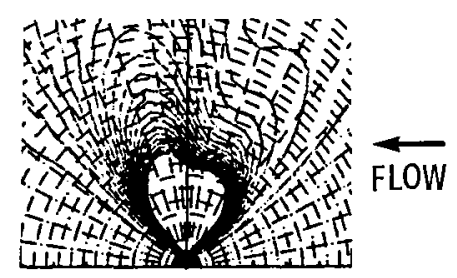

(a) FREE FIELD

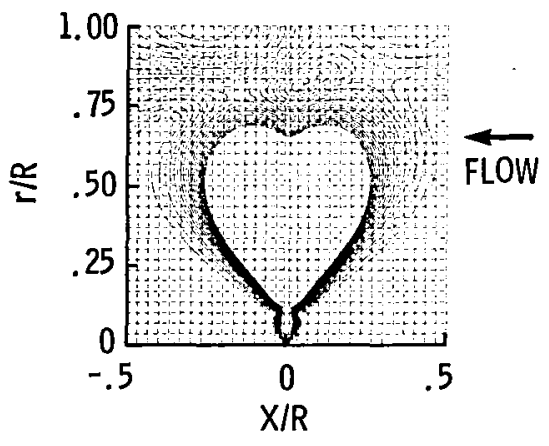

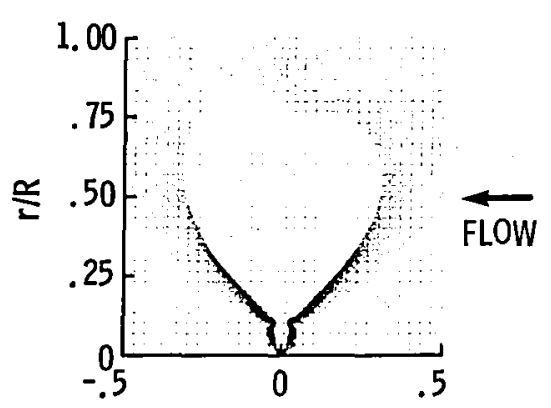

(c) $A=0.5+i 0.0$

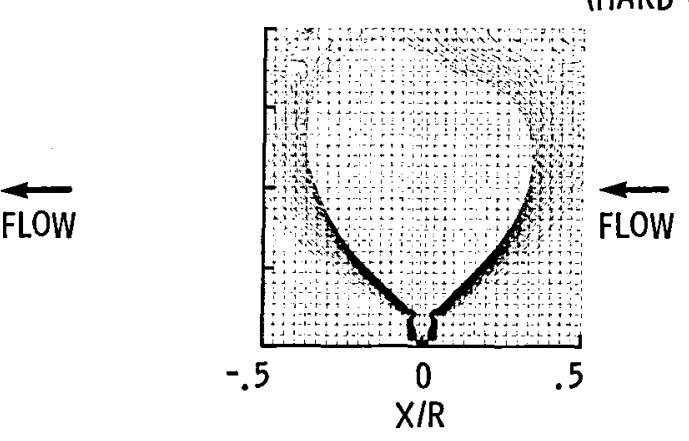

(d) $A=0.91+i 0.0$

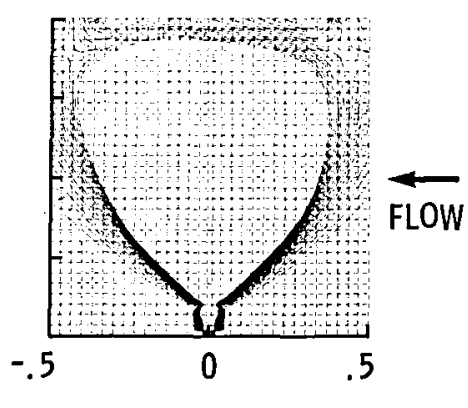

(e) $A=2.0+i 0.0$

Figure 11. - Directivity for an eight bladed propeller in a duct with a wall to propeller radius of 2 for a variety of lining admittances; $m=8, N=8, \eta_{P}=5.69$ (propeller), $\eta_{R}=11.38$ (tunnel), $M=-0.2, n=1$ (first harmonic. 


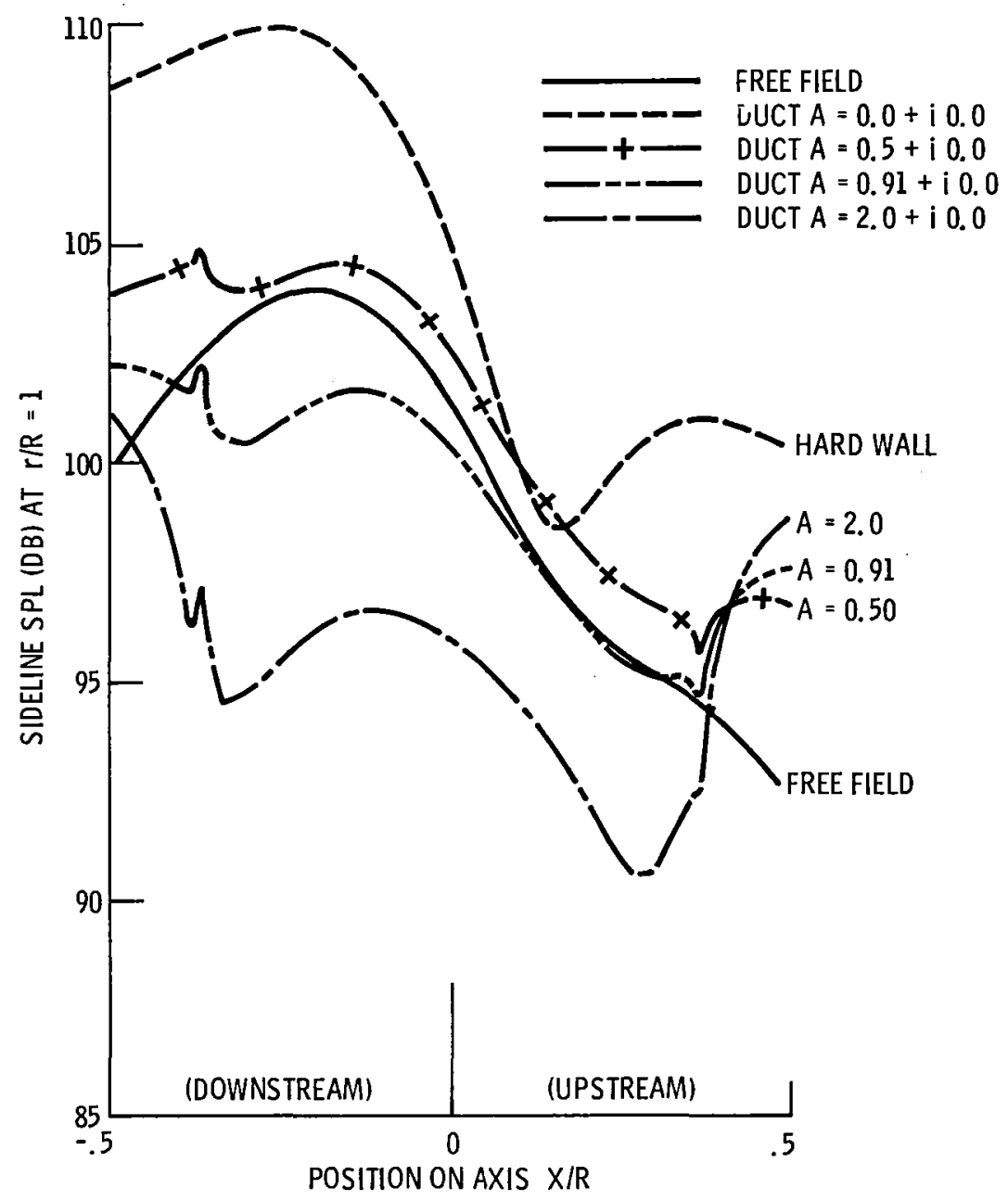

Figure 12. - Sideline directivity at tunnel radius of two propeller diameters $\left(r / R_{p}=2\right.$ or $\left.r / R=1\right)$ comparing the propeller in the free field (solid line) and in the tunnel with several lining admittances; $m=8, N=8$, $\eta_{P}=5.69$ (propeller), $\eta_{R}=11.38$ (tunnel), $M=-0.2$, $\mathrm{n}=1$ (first harmonic). 


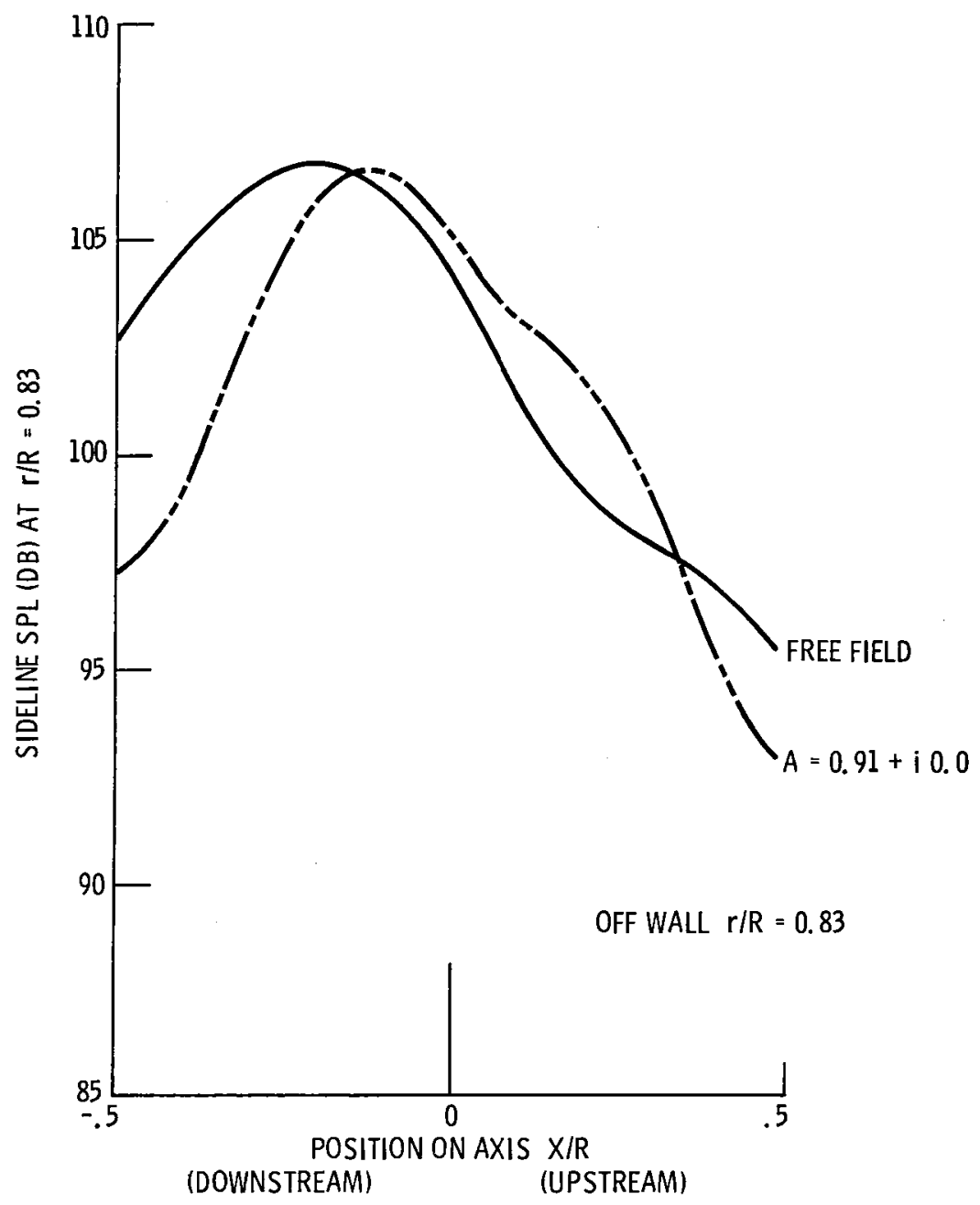

Figure 13. - Sideline directivity at tunnel radius ratio $r / R=0.83$ (or $r / R_{p}=1.667$ ) comparing the propeller in the free field (solid line) and in the tunnel for $A=0.91+i 0.0, m=8, N=8, \eta_{P}=5.69$ (propeller), $\eta_{R}=11.38$ (tunnel), $M=-0.2, n=1$ (first harmonic). 


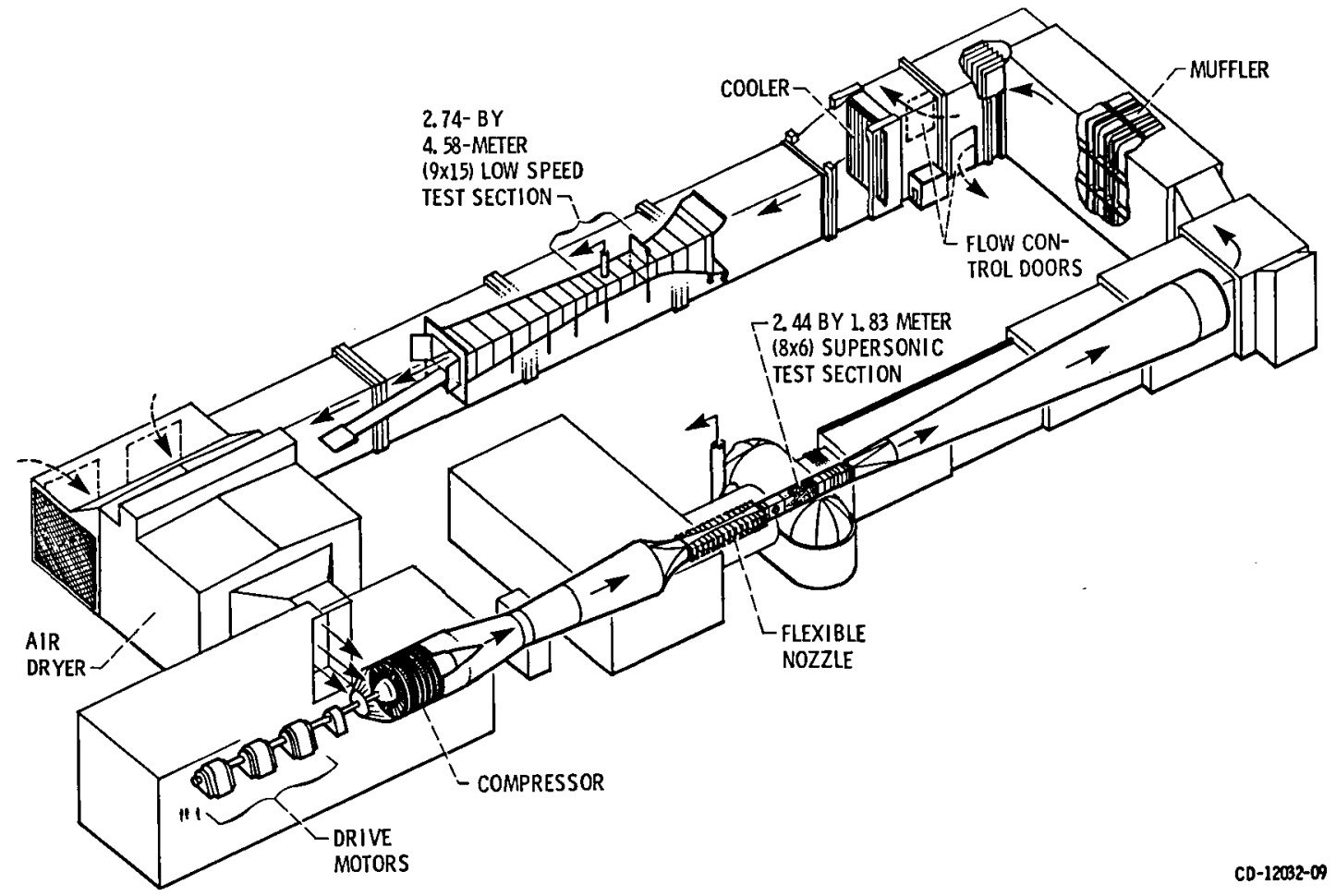

Figure 14. - NASA Lewis 8- by 6-foot and 9- by 15 -foot wind tunnels.

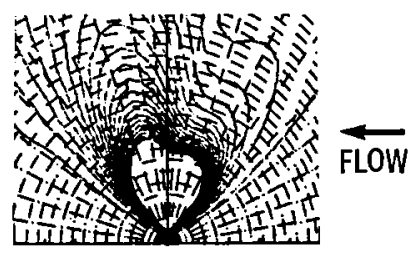

(a) FREE FIELD

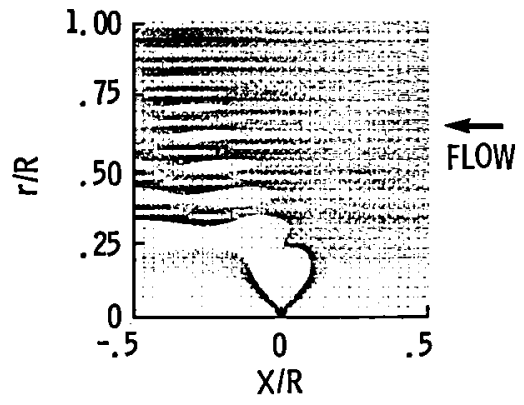

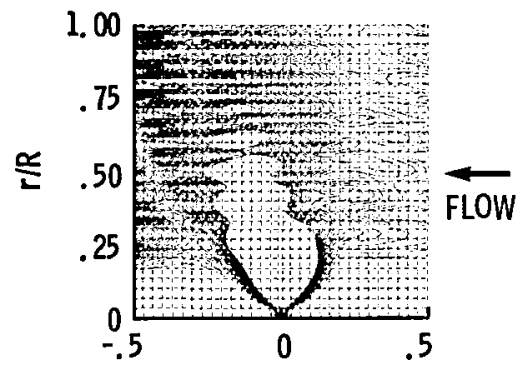

(c) $A=0.5+i 0.0$

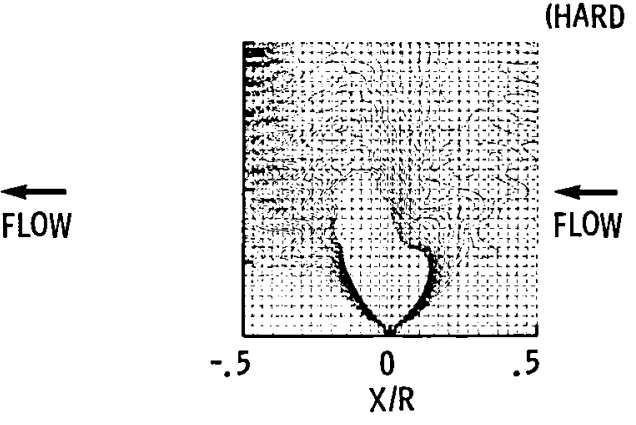

(d) $A=0.91+i 0.0$

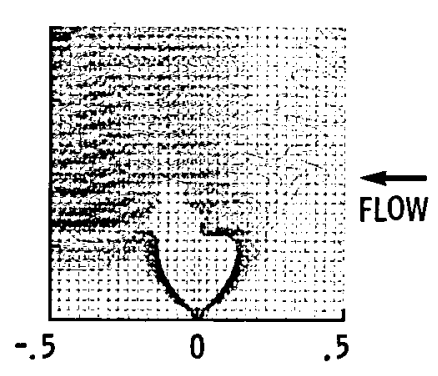

(e) $A=2.0+i 0.0$

Figure 15. - Directivity for an eight bladed propeller in a duct with a wall to propeller radius of 6 for a variety of lining admittances; $m=8, N=8, \eta_{P}=5.69$ (propeller), $\eta_{R}=34.13$ (tunnel), $M=-0.2, n=1$ (first harmonic.) 


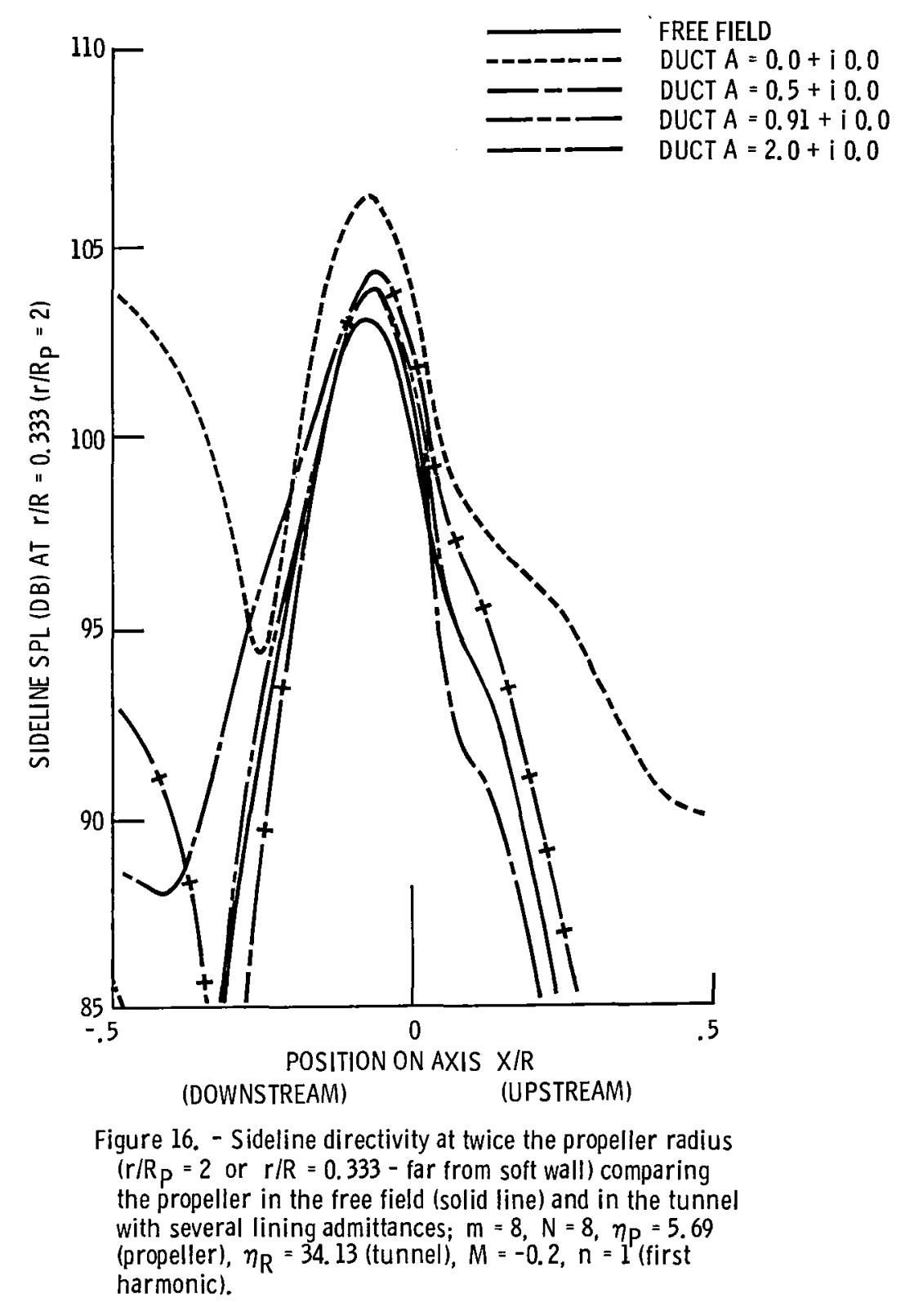




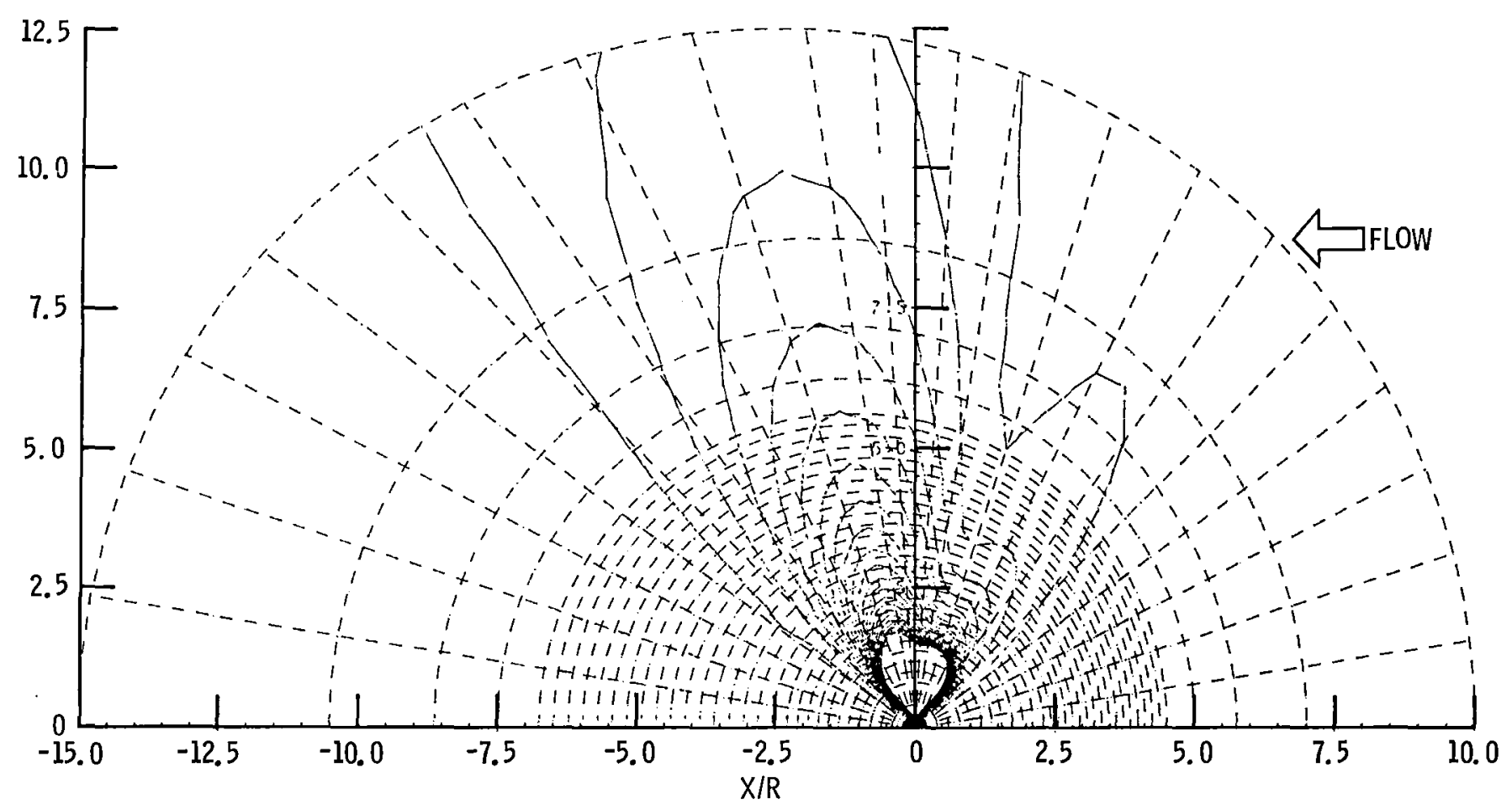

Figure 17. - Free field directivity for an eight-bladed propeller in a slow (landing) mean flow; $m=8, N=8, \eta_{P}=5.69$, $M=-0.2, n=1$ (first harmonic). 


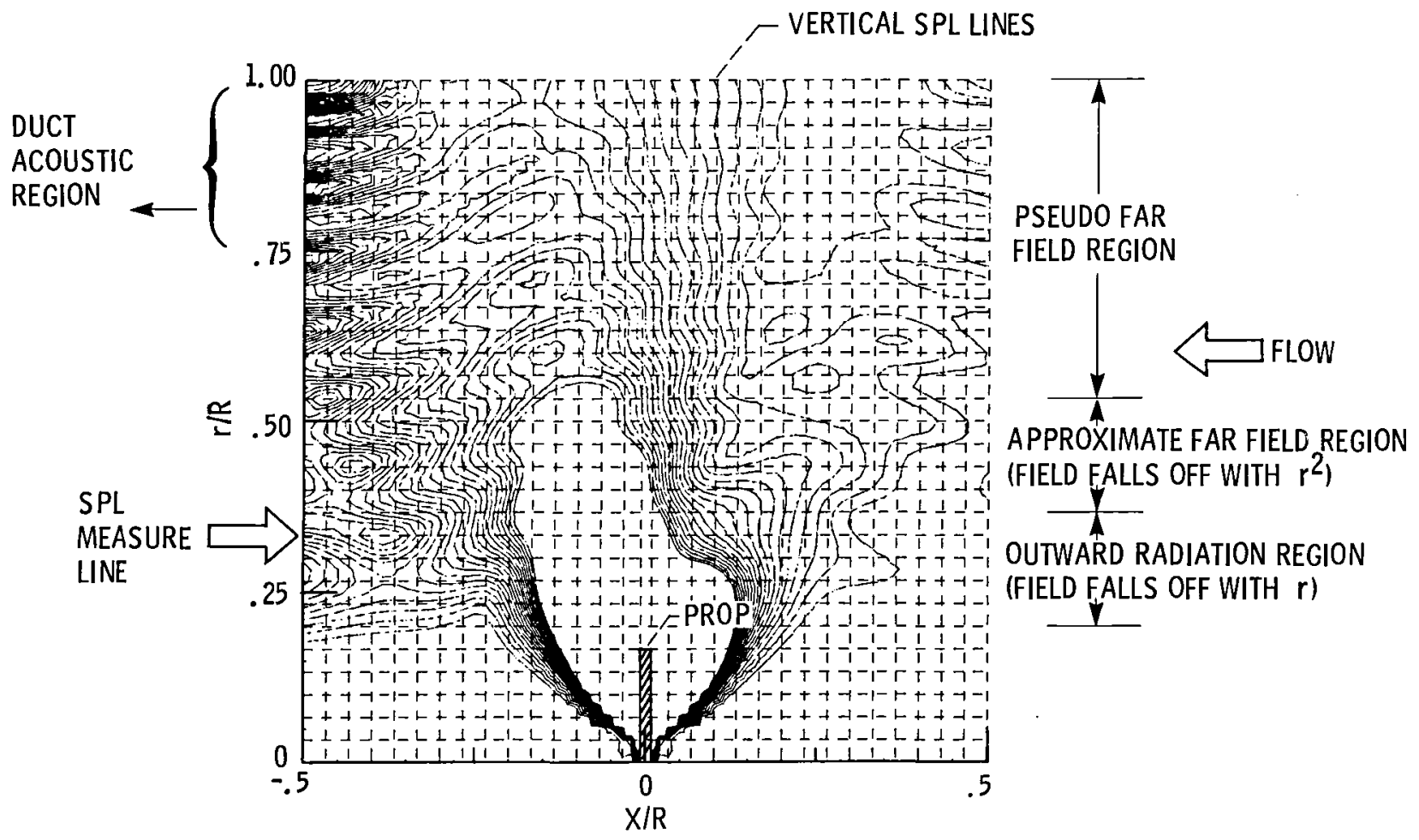

Figure 18. - Directivity for an eight bladed propeller in a duct with a wall to propeller radius of 6 for a wall admittance of $0.91+i 0.0, m=8, N=8, \eta_{p}=5.69$ (propeller), $\eta_{R}=34.13$ (tunnel), $M=-0.2, n=1$ (first harmonic). 


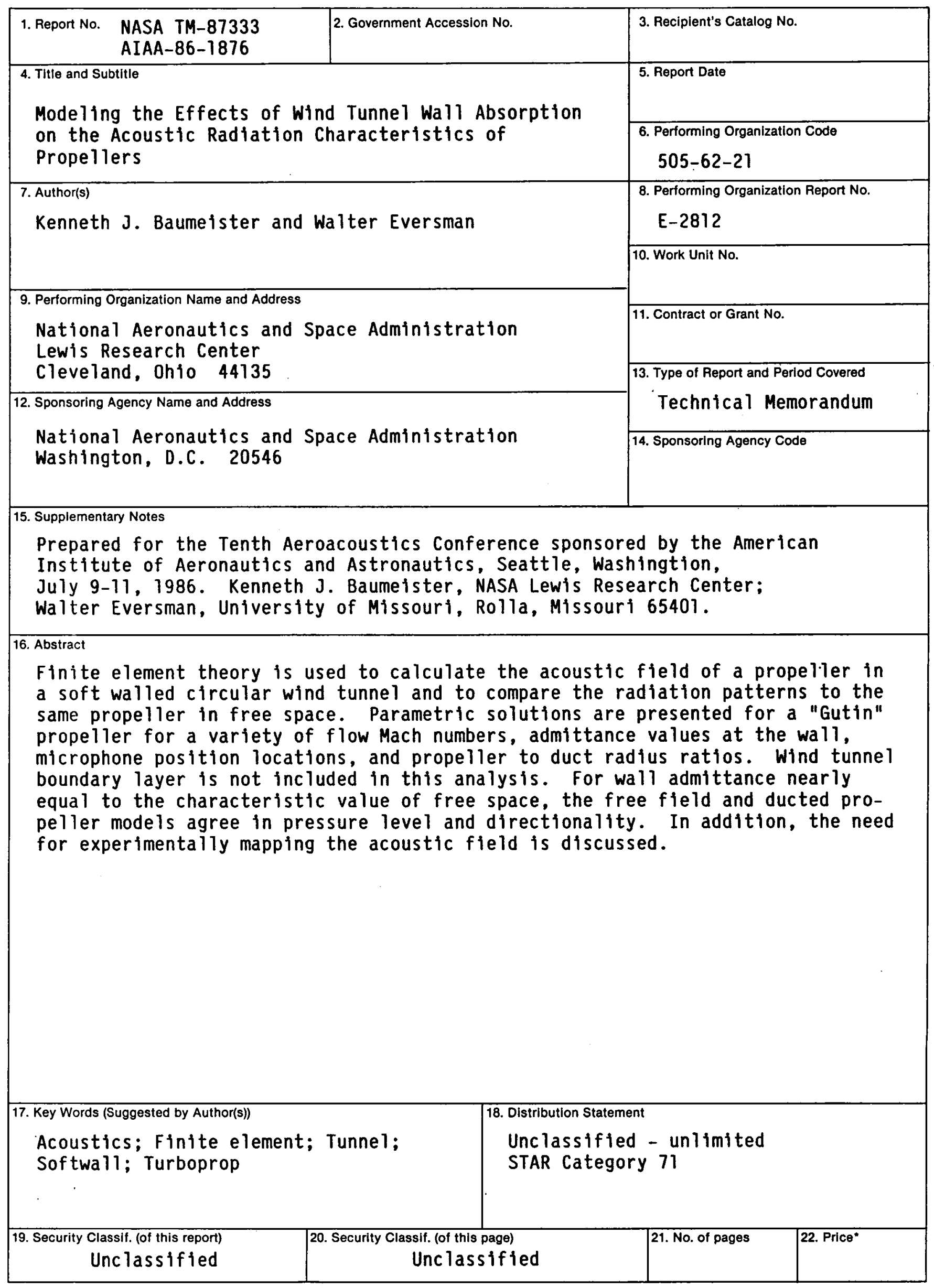


End of Document 